\title{
Repeated multiple intracranial hemorrhages induced by cardiac myxoma mimicking cavernous angiomas: a case report
}

\author{
Kai Quan, Jianping Song ${ }^{*}$, Wei Zhu, Liang Chen, Zhiguang Pan, Peiliang Li and Ying Mao
}

\begin{abstract}
Background: Repeated intracranial hemorrhages caused by cardiac myxoma is very rare. It is essential for physicians to be aware of such uncommon clinical feature of myxoma.

Case presentation: We report a-49-year-old female patient complained of repeated multiple intracranial hemorrhages, with no sign of cardiac dysfunction or cerebral infarction before admission. Cavernous angioma (CA) was misdiagnosded due to the clinical and magnetic resonance (MR) presentation. A sudden ischemic event after admission led to the finding of a left atrial myxoma.

Conclusions: Repeated intracranial hemorrhages can be the early and primary clinical presentation of cardiac myxoma, probably caused by its metastasis, without obvious ischemic stroke or cardiac symptoms.
\end{abstract}

Keywords: Cavernous angioma, Intracranial hemorrhage, Ischemic stroke, Myxoma

\section{Background}

Cardiac dysfunction often present as the primary complain of atrial myxoma and patients may end up with sudden death [12]. Without cardiac symptoms, the variety of clinical features, involving neurologic or cutaneous symptoms, could make the diagnosis of atrial myxoma very difficult $[4,6,14]$. Even up to $10 \%$ of patients with atrial myxoma may not present with any symptoms $[4,8,12,16]$.

Cardiac myxoma may be found incidentally while screening the source of intracranial embolus. However, physicians rarely include atrial myxomas on differential diagnosis list for intracranial hemorrhages. Thereby, we would like to present a case of cardiac myxoma inducing multiple hemorrhagic intracranial lesions mimicking CAs.

\section{Case presentation}

A-49-year-old female patient was admitted to local emergency department in November 2012 and August 2013, suffered from acute headache and dizziness, without any neurologic deficit. No history of hypertension or

\footnotetext{
* Correspondence: Neurosurgerysong@foxmail.com

Department of Neurosurgery, Huashan Hospital of Fudan University, No. 12 Wulumuqi Zhong Road 200040, Shanghai, China
}

familiar CAs was recorded. In both time, Emergency head Computer Tomography (CT) has showed three hemorrhagic intracranial lesions in the left parietoccipital lobe (Fig. 1). Routine head MR scan also showed three small hemorrhagic lesions, with low signal on T1-weighted and high signal on T2-weighted images, without marked enhancement. A significant hypointese ring outside the lesion represents hemosiderosis, supporting diagnosis of CA. No acute infarction was detected in diffusion weighted imaging (DWI) (Fig. 1). Considering the good recovery afterwards and the risk of operation, she accepted the wait-and-see treatment.

In February 2014, she experienced recurrent symptoms. This time, she decided to accept intracranial lesions resection. She was admitted to our hospital in March 2014 for futher examination and surgical plan without any complaints of head or chest discomfort at that time.

In the next morning of hospitalization, she lost consciousness suddenly. Emergent $\mathrm{CT}$ indicated the third episode of hemorrhagic within the lesions. However, after regaining her consciousness, complete contralateral paralysis, facial palsy and aphasia -without prior seizure-, occured. Those symptoms could not be explained by the lesions. 


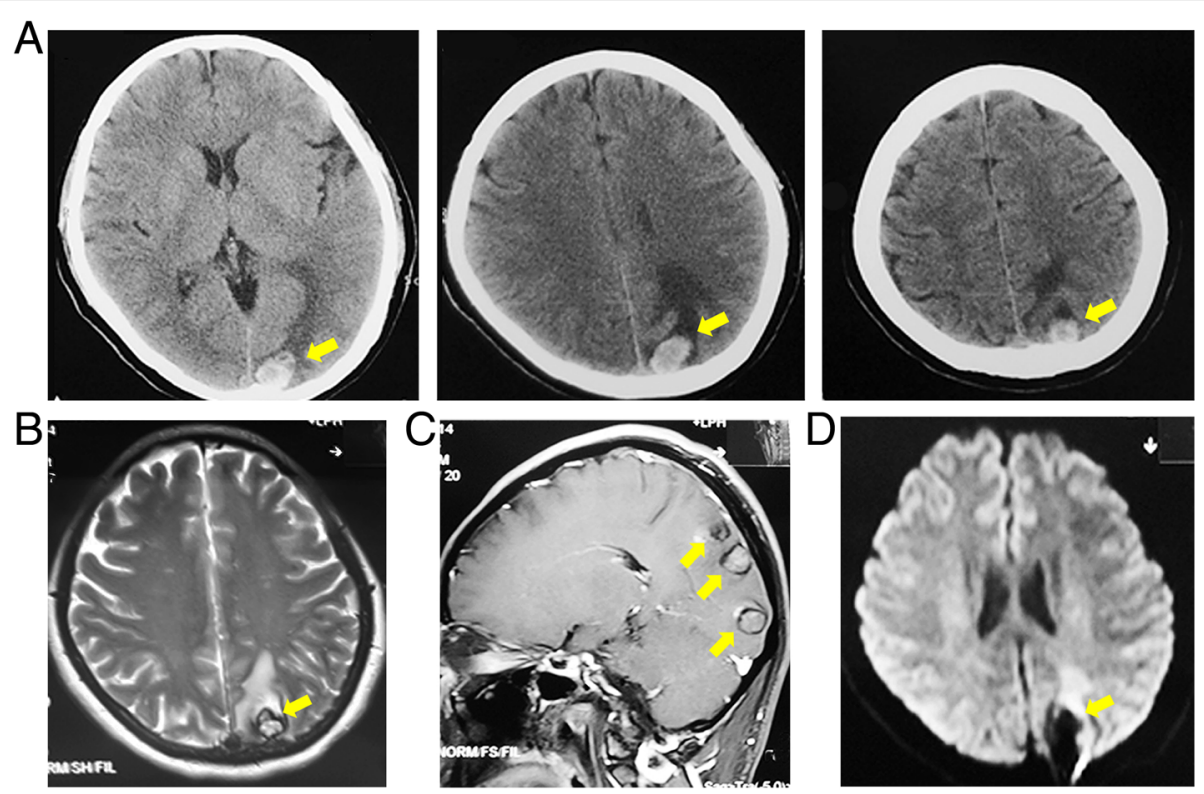

Fig. 1 Multiple intracranial hemorrhages detected in head CT scans in this case (Yellow arrows) (a). Head MR scans illustrated hyperintensive lesions on T2-weighted images, with peripheral hypointensities (Yellow arrows) (b). No marked enhancement was detected in these three lesions (Yellow arrows) (c). Lesions showed low signals in DWI (Yellow arrows), and no other abnormal signals were found (d)

Supportive treatment and intensive monitoring was initiated for her, and she did not deteriorate. The Susceptibility Weighted Imaging (SWI) revealed numerous hypointense foci, in addition to the first known lesions, mimicking CAs in the brain and indicating previous hemorrhages (Fig. 2). Additional DWI detected multiple minor acute infarction throughout the left hemisphere, including left basal ganglia, thalamus and cortex (Fig. 2).

To identify the cause of embolism, a MR angiography and echocardiogram was performed. No abnormalities were detected in the large intracranial arteries by MR angiography, but a suspicious small aneurysm next to one of the CA like lesions (Fig. 2). Echocardiogram
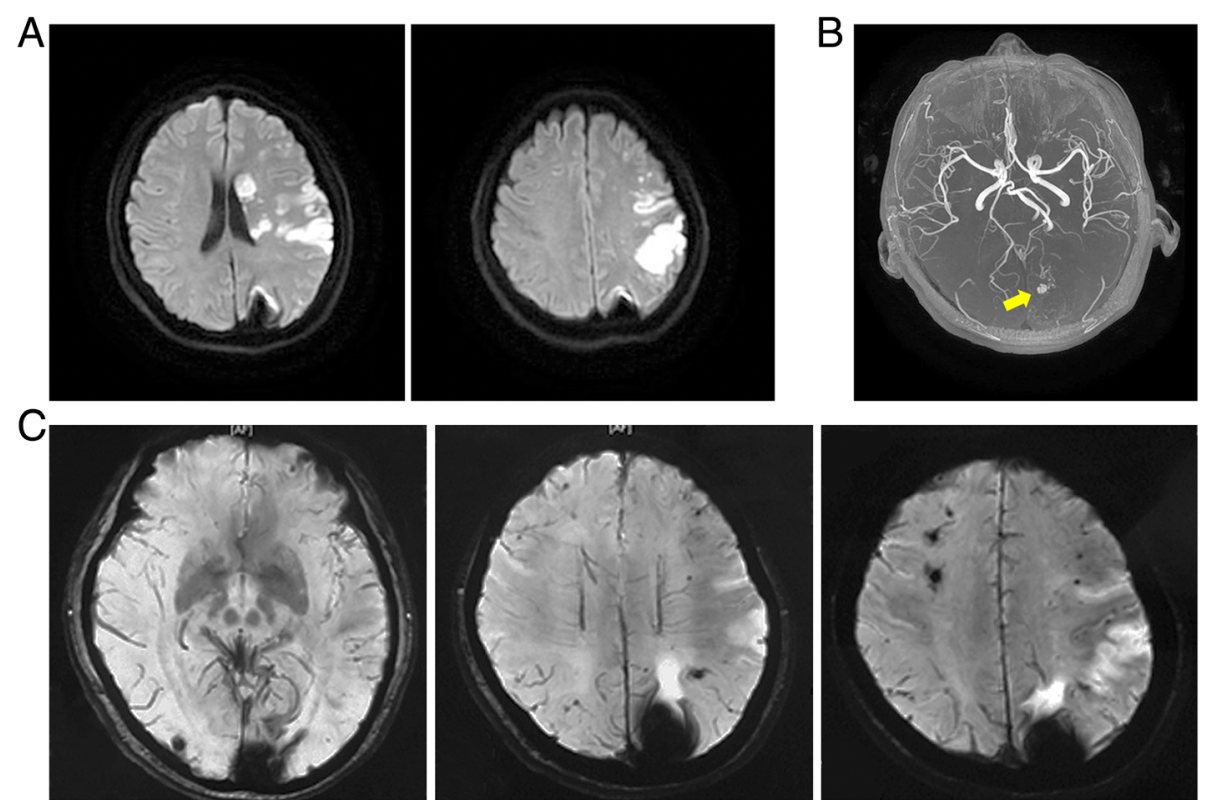

Fig. 2 After the onset of apsychia, multiple high intensive signals appeared in DWI throughout the left hemisphere (a). A suspicious aneurysm was detected next to the lesion (b). SWI scan revealed numerous hypointense foci in the whole brain (c) 
demonstrated a $55 \% 28 \mathrm{~mm}$ left atrial myxoma, blocking blood flow through the mitral valve. After several days of conservative treatment and rehabilitation, she was able to speak slowly and the muscle strength of the right limb improved to Grade 4.

During harboring the atrial myxoma, the risk of perioperative complications, such as acute cardiac dysfunction, ischemic stroke or sudden death, are highly increased. Thus, we cancelled our surgical plan and consulted to the department of cardiac surgery. The patient was scheduled for atrial myxoma removal after recovery. The post-operative pathology confirmed the diagnosis of cardiac myxoma (Fig. 3).

\section{Discussion}

Classic presenting manifestations of cardiac myxoma include constitutional, obstructive, and embolic symptoms [5]. Cerebral ischemia may be the most significant complaint despite cardiac dysfunction symptoms In patients with left atrial myxoma, $11 \%-45 \%$ of ischemic strokes could be induced by a small emboli which is detached from the myxoma [5, 6]. Cerebral infarction was the most common manifestation in up to $89 \%$ among myxoma patients with neurologic symptoms [6]. Therefore, myxoma is less likely to be ignored in case of repeated ischemic stroke.

However, only a small fraction of patients with myxoma was found due to intracranial hemorrhages $[6,8]$. Single hematoma or multiple scattered microbleeds, also subarachnoid hemorrhage might be present. These hemorrhages are usually related to previous cerebral infarction caused by myxoma $[6,8,14,16]$. However, it is quite rare that repeated intracranial hemorrhages without definitive history of ischemic stroke or cardiac symptoms, could be the first and primary clinical presentation. The radiological finding in routine MR imaging, especially the hemosiderin signal around the

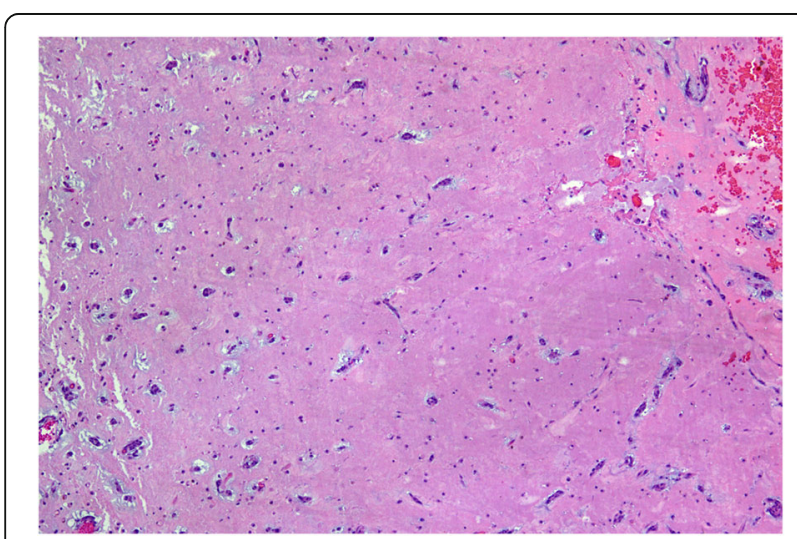

Fig. 3 The pathological appearances of resected cardiac myxoma demonstrated myxiod stroma containing round or fusiform pleomorphic cells and inflammatory cells (HE staining, original magnification $\times 5$ ) lesions, strongly support the diagnosis of CA. Therefore, multiple CAs was misdiagnosed according to the clinical and MR features before admission.

Even SWI features of this patient imitate CAs. It is well known that SWI is crucial to diagnose multiple CAs, because it is very sensitive in detecting previous microbleeds according to the remaining hemosiderin with ferromagnetic composition [3, 10]. But in this case, SWI might be confusing. The presence of hemorrhagic lesions combined with numerous microbleeds in SWI indicated multiple CAs. However, considering embolic stroke afterwards, the visible microbleeds is also likely to be caused by unnoticed previous minor asymptomatic lacunar stroke, probably due to micro embolus detached from cardiac myxoma.

Embolic stroke after admission led us to diagnose cardiac myxoma eventually. But how did myxoma cause repeated hemorrhages without an obvious ischemic stroke before admission? It was reported that myxomatous aneurysms might be an alternative source of bleeding $[7,13,16]$. About $1 / 3$ of patients treated for atrial myxoma may present with a cerebral aneurysm [17]. In this case, the head MR angiography showed a suspicious aneurysm, but it is not responsible for all hemorrhages apparently. After reviewing previous literatures, it seems that intracranial hemorrhages of atrial myxoma are relatively rare, and may be repeated multiple hemorrhages, some bleeding co-exist with subarachnoid hemorrhage without angiographic evidence of aneurysms $[2,13]$. Another explanation is that cardiac myxoma may break down and travel along the blood stream, and the metastasis can bleed inside [11]. In this case, we believe that these intracranial hemorrhagic lesions may be mainly myxoma metastasis.

Although in this case, the patient did not have irreversible poor prognosis after repeated intracranial hemorrhages and ischemic event after admission, it should alert the clinicians to pay more attention to such various clinical features of myxoma. The repeated hemorrhagic signals in CT due to myxoma may hinder the clinicians to make a correct diagnosis of cerebral ischemia. However, if mxyoma cannot be detected in time, the hemostatic treatment according to the $\mathrm{CT}$ presentation may exacerbate cerebral ischemia and catastrophic results, such as acute cardiac dysfunction, ischemic stroke or sudden death, during anesthesia or around the perioperative period. Therefore, cardiac examination, such as echocardiography, should be performed as an alternative screening tool for cardiac myxoma, in case of unclear repeated multiple intracranial hemorrhages, markedly unmatched symptoms. Especially with unmatched symptoms.

There are no definite therapeutic guidelines about further intervention for this disease. Resection of the 
cardiac myxoma firstly is useful to eliminate the original sources of metastastic lesion, however which cannot completely abolish the risk of delayed cerebral aneurysm formation [17]. However, because cerebral aneurysm from cardiac myxoma are probably multiple and rarely associated with intracranial hemorrhage, most of the patients can be managed conservatively [18]. Antifibrinolytic drug such as aminomethylbenzoic acid can be used to prevent rebleeding [13]. If severe intracranial hemorrhage due to myxoma do occur, excision of large intracranial hematoma and metastatic lesions remains supportive, and is useful in eliminating early neurologic symptoms [13]. For this case, we believe that further intracranial lesions resection is more suitable than consevative therapy. Some authors also suggest that chemotherapy in combination with low-dose radiation for multiple lesions, but there are only four reported cases that received 25-60Gy brain radiation in previous studies,and one of these patients died of enlarging intracranial masses $[1,9,13,15]$.

\section{Conclusions}

In summary, the neurosurgical community should be aware that repeated multiple intracranial hemorrhages can be the primary cerebral presentation of cardiac myxoma, probably due to myxoma metastasis, despite no significant ischemic stroke for a period. Routine CT, MR and SWI of intracranial hemorrhagic lesions induced by cardiac myxoma may mimics CAs. Therefore, cardiac examination should be performed in case of unusual multiple intracranial hemorrhages, in order to determine the presence of cardiac myxoma.

\section{Abbreviations}

CA: Cavernous angioma; CT: Computer tomography; DWl: Diffusion weighted imaging; MR: Magnetic resonance; SWI: Susceptibility weighted imaging

\section{Acknowledgements}

The authors sincerely thank Dr. zakaria shahab for editorial assistance, and this study was sponsored by the National Natural Science Foundation of China (Nos. 81400962 (JS),81571102(WZ)).

\section{Availability of data and materials}

The manuscript does not include any new software, databases or all relevant raw data.

\section{Authors' contributions}

JS and YM conceived the project and designed the study. KQ collected patient's data. WZ and LC provided technical assistance in the study. ZP and PL prepared the illustration. JS and KQ analyzed data and wrote the paper. All authors approved the paper for the submission.

\section{Competing interests}

The authors have no personal financial or institutional interest in any of the drugs, materials, or devices described in this article.

\section{Consent for publication}

Informed consent was obtained from patient for the publication of this report and any accompanying images. A copy of the written consent is available for review upon request.

\section{Ethics approval and consent to participate}

The manuscript has had ethics approval and consent to participate by medical ethics committee of huashan hospital.

Received: 2 June 2016 Accepted: 8 December 2016

Published online: 13 January 2017

\section{References}

1. Altundag MB, Ertas G, Ucer AR, Durmus S, Abanuz H, Calikoglu T, et al. Brain metastasis of cardiac myxoma: case report and review of the literature. J Neurooncol. 2005;75:181-4.

2. Brinjikji W, Morris JM, Brown RD, Thielen KR, Wald JT, Giannini C, et al. Neuroimaging findings in cardiac myxoma patients: a single-center case series of 47 patients. Cerebrovasc Dis. 2015;40:35-44.

3. de Souza JM, Domingues RC, Cruz Jr LC, Domingues FS, lasbeck T, Gasparetto EL. Susceptibility-weighted imaging for the evaluation of patients with familial cerebral cavernous malformations: a comparison with t2-weighted fast spin-echo and gradient-echo sequences. AJNR Am J Neuroradiol. 2008:29:154-8.

4. Garcia FVMJ, Sanz-Sanchez T, Aragues M, Blasco A, Fraga J, Garcia-Diez A. Cutaneous embolization of cardiac myxoma. Br J Dermatol. 2002;147:379-82.

5. Knepper LE, Biller J, Adams Jr HP, Bruno A. Neurologic manifestations of atrial myxoma. A 12-year experience and review. Stroke. 1988;19:1435-40.

6. Lee VH, Connolly HM, Brown Jr RD. Central nervous system manifestations of cardiac myxoma. Arch Neurol. 2007;64:1115-20.

7. Li Q, Shang H, Zhou D, Liu R, He L, Zheng H. Repeated embolism and multiple aneurysms: central nervous system manifestations of cardiac myxoma. Eur J Neurol. 2008;15:e112-3.

8. Michael AS, Mikhael MA, Christ M. Myxoma of the heart presenting with recurrent episodes of hemorrhagic cerebral infarction: MR findings. J Comput Assist Tomogr. 1989;13:123-5.

9. Moiyadi AV, Moiyadi AA, Sampath S, Kalpana SR, Mahadevan A, Shankar SK, et al. Intracranial metastasis from a glandular variant of atrial myxoma. Acta Neurochir (Wien). 2007;149:1157-62.

10. Nandigam RN, Viswanathan A, Delgado P, Skehan ME, Smith EE, Rosand J, et al. MR imaging detection of cerebral microbleeds: effect of susceptibilityweighted imaging, section thickness, and field strength. AJNR Am J Neuroradiol. 2009;30:338-43.

11. Park J, Song JM, Shin E, Jung SH, Kim DH, Kang DH, et al. Cystic cardiac mass in the left atrium: hemorrhage in myxoma. Circulation. 2011;123:e368-9.

12. Pinede $L$, Duhaut $P$, Loire R. Clinical presentation of left atrial cardiac myxoma. A series of 112 consecutive cases. Medicine (Baltimore). 2001;80:159-72.

13. Raza E, Kamal AK. Recurrent non-aneurysmal, metastatic intraparenchymal haemorrhages following resection of atrial myxoma - case report and literature review. BMJ Case Rep. 2012.

14. Tamuleviciute E, Taeshineetanakul P, Terbrugge K, Krings T. Myxomatous aneurysms: a case report and literature review. Interv Neuroradiol. 2011;17:188-94.

15. Todo T, Usui M, Nagashima K. Cerebral metastasis of malignant cardiac myxoma. Surg Neurol. 1992;37:374-9.

16. Vanacker $\mathrm{P}$, Nelissen N, Van Laere K, Thijs VN. Images in neurology. Scattered cerebral microbleeds due to cardiac myxoma. Arch Neurol. 2009:66:796-7.

17. Vigano S, Papini GD, Cotticelli B, Valvassori L, Frigiola A, Menicanti L, et al. Prevalence of cerebral aneurysms in patients treated for left cardiac myxoma: a prospective study. Clin Radiol. 2013;68:e624-8.

18. Zheng J, Zhang J. Neoplastic cerebral aneurysm from metastatic tumor: a systematic review of clinical and treatment characteristics. Clin Neurol Neurosurg. 2015;128:107-11. 